\title{
A New Color Image Segmentation Algorithm based on Watershed Transformation
}

\author{
Marat Kazanov \\ Computer Science Department, Institute for Systems Analysis Academy of Science of Russia \\ mkazanov@mail.ru
}

\begin{abstract}
A new color segmentation method is presented in this paper. The method is specified for color images that have both large and small objects, and objects with both step and ramp edges. Scanned pages of color magazines and newspapers are the examples of this kind of images. Watershed transformation algorithm is the basis of the proposed method. Our method incorporates the original multi-scale analysis that allows to segment edges of different slope. This analysis uses fine-to-coarse strategy and prevents the already detected sharp edges from smoothing while moving to coarser scales. In the same time the introduced algorithm allows to detect ramp edges successfully at coarse scales. For fine scales we propose a special gradient operator and a modification of watershed transformation for small objects segmentation.
\end{abstract}

\section{Introduction}

Today color image segmentation is a rapidly developing area of digital image processing [1]. Color image segmentation attracts more attention due to the increasing computational potentialities of personal computers and possibility of their usage for color image processing. Nevertheless, this problem remains less investigated than segmentation of grayscale images. There are many application areas for color image segmentation, one of them is OCR (Optical Character Recognition) systems. Segmentation is the first stage in operation of OCR systems. OCR systems usually apply binarization methods for segmentation of grayscale images. However, application of these methods for color images is impossible in general. For this reason, development of segmentation methods for color images is a very topical problem.

The known color segmentation approaches are based on monochrome segmentation methods modified for using color information [1]. The major segmentation ap- proaches for monochrome images are histogram thresholding, edge detection, region-based methods, fuzzy techniques, etc. Each of these methods has its particular advantages and disadvantages, and therefore various methods are preferred in each specific case. For example, histogram thresholding has low computational complexity and can be used for a wide class of images, but it ignores spatial information [1]; region-based methods are noise immune, but they require selection of seed regions [1].

Our objective was to develop a color segmentation method for application in OCR systems. Typical images to be worked at are scanned pages of color magazines and newspapers. As a rule, objects to be segmented on such images (text, pictures, titles, blocks of articles, etc.) have clearly defined edges. Parts of pictures and the background usually have less distinct edges, but their segmentation is less important. The above mentioned has led us to the conclusion that edge-based methods are preferable for segmentation of scanned images from color magazines and newspapers. In our work we use watershed transformation [2]the popular edge-based segmentation method.

Advantages of watershed transformation are wellknown [3]: it allows to detect objects' boundaries precisely; this method always produces continuous segmentation boundaries that help to avoid post-processing, such as edge linking, which is usual for other methods. Another advantage is that there exist many fast and computationally efficient algorithms of watershed transformation [4],[5]. However, there are two problems to be solved: (1) inner area of small objects, such as letters or symbols, is often too small to form basins used in the watershed transformation algorithm (2) it is impossible to detect boundaries of objects with both ramp and step edges using one-scale gradient image only. We tried to solve these problems and to develop an efficient segmentation method for color images from magazines and newspapers, as well as others images of this kind.

Section 2 reviews our approaches for multi-scale segmentation and segmentation of small objects, the proposed algorithm on the whole is briefly described. Ex- 
perimental results are presented in section 3 and conclusion is given in section 4 .

\section{Proposed method}

As was mentioned above, the proposed method is based on the watershed transformation algorithm. As a rule, this algorithm is applied not to the original, but to the gradient image. Application of a gradient operator at each point of the original image constructs the gradient image. Gradient operator for grayscale images is based on the intensity differences at points with assigned weight coefficients. At color images difference in color can be used instead of intensity difference. This color difference can be measured as distance in a particular color space. There are many color spaces: RGB, Lab, CIELab, etc. Usage of any of the mentioned color spaces has particular advantages and disadvantages. Choice of color space is not discussed in this paper; for the sake of simplicity we use RGB color space.

The proposed method includes two new approaches: our approach to the multiscale analysis and approach to the segmentation of small objects. It is known that the best segmentation of different objects can be executed at different scales of a gradient image [6]. The proposed multiscale algorithm allows to segment different objects combining information from different scales. Algorithm of small objects segmentation allows to segment objects that can not be segmented by traditional watershed algorithm due to their small size.

These proposed approaches would be discussed in detail in sections 2.1-2.2. Description of the proposed method will be presented in section 2.3.

\subsection{Multiscale analysis}

In many cases, the original image contains objects with step edges as well as objects with ramp edges. Gradient image can be constructed using different gradient operator masks that vary in size. Objects with step edges are successfully segmented at gradient images of fine scale, i.e. images constructed using masks of small size. On coarser scales step edges blur and therefore can be segmented inaccurately. Objects with ramp edges are successfully segmented at coarse scales, but at finer scales ramp edges have insufficient intensity for segmentation. Consequently, successfully segmentation of both step and ramp edges demands gradient image analysis of different scales.

The proposed multiscale approach is based on a fineto-coarse tracking strategy. Each step of the proposed algorithm is the analysis of a one-scale gradient image. The first step includes gradient image construction and edge detection. On next stages gradient image is constructed, a)

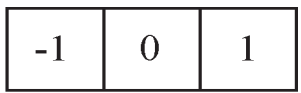

b)

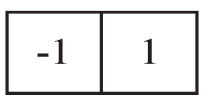

Figure 1. a) 3-pixels first-order derivative mask.

b) 2-pixels first-order derivative mask.

taking account of positions of edges detected at previous steps. Let's describe construction of a s-scale gradient image: gradient operator mask is applied at each point of the original image excluding points that belong to sneighbourhood of edge pixels. This rule of the gradient image construction prevents step edges detected at small scales from blurring. In the same time, this rule does not prevent the detection of ramp edges at coarse scales. The proposed approach is described in detail in [7].

\subsection{Segmentation of small objects.}

Watershed algorithm gives acceptable results not for all object types. Successful segmentation is impossible for objects' details or small objects sized less than the gradient operator mask. This is conditioned by the shift of the small objects' boundaries during the construction of the gradient image [8]. For example, correct detection of edges with the help of the traditional 3x3 Roberts [9] or Prewitt [10] masks is impossible if the edges of objects are sized less than 3 pixels. In practice we can find small objects very often. Letter width in the texts on scanned from color newspapers or magazines with resolution 100 pixels/inch usually equals 2-3 pixels. Our objective was to develop a watershed transformation based approach allowing to segment objects sized up to 1 pixel.

The proposed method consists of applying a special gradient operator and a modification of watershed transformation. Application of the special gradient operator allows to approximate values of the first-order derivative not only in pixels of the original image, but also between pixels. The proposed special gradient operator is application of the two smallest masks to the original image. For simplicity we will first consider the gradient operator in a onedimensional case, and then extend it to two dimensions.

We will use two smallest masks that approximate the first-order derivative of the image. Figure 1(a) shows the mask we will use for estimation of values of the derivative at each point of the image. Figure 1(b) shows the mask we will use for estimation of values of the derivative between image points. Let us define our approximation of the first-order derivative operator formally. Let $I(x):=I(i), i=0,1, . . N$ be an original image profile. Let 


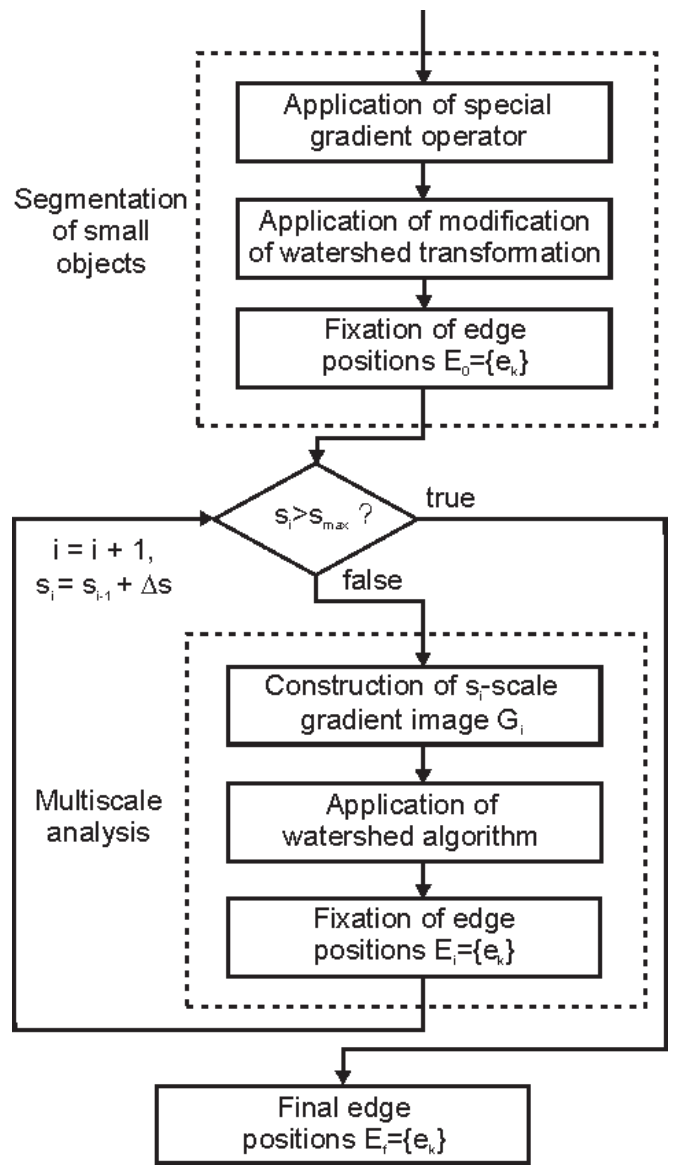

Figure 2. Scheme of proposed algorithm

$G(x):=G(j), j=0,1, . .2 N$ be our approximation of the firstorder derivative of the image. Resolution of $G(x)$ increases doubly in comparison with the resolution of the original image profile $I(x)$. We define $G(x)$ as:

$G(x)=\left\{\begin{array}{l}\text { even } x:[I(x / 2+1)-I(x / 2-1)] / 2 \\ \text { odd } x: I((x+1) / 2)-I((x-1) / 2)\end{array}\right.$

Thus, we introduce now our special gradient operator that consists of the combination of gradient masks sized 3 and 2 pixels.

Let us extend the above mentioned operator to a twodimensional case. As in one-dimensional case we will compute values of the gradient not only in pixels of original image, but also between pixels. The computation of partial derivatives $\mathrm{df} / \mathrm{dx}$ and $\mathrm{df} / \mathrm{dy}$ is required for the estimation of gradient amplitude. The computation of partial derivatives can be executed as in a one-dimensional case. Nevertheless, it is impossible to compute partial derivatives using equation (1) for all types of subpixel positions. It is impossible in the positions, where the vertical or the horizontal line crossing this position does not intersect image pixels. A larger mask covering image pixels is be necessary for computation of partial deriva- a)

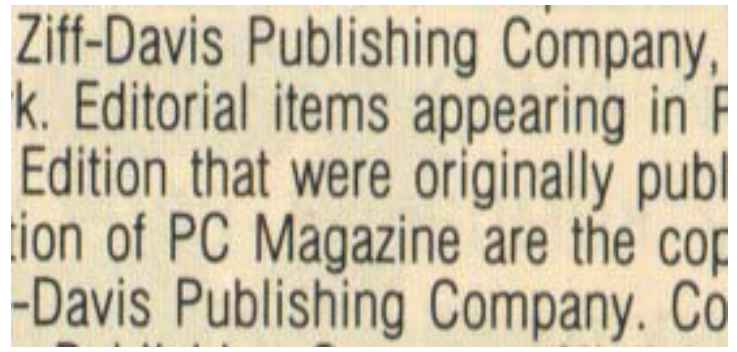

b) Ziff-Davis Publishing Company, $k$. Editorial items appearing in $F$ Editıon that were originally publ ion of PC Magazine are the $\mathrm{Cof}$ -Davis Publishing Company. Co

Figure 3. a) Original image with resolution 306×155

b) Result of segmentation using proposed method

tives in such subpixels positions. Such mask would give a coarser estimation of the value of the first-order derivative. Since our original goal is to detect the positions of object's boundaries as accurate as possible, we decided not to compute all the components of the image gradient between pixels. Instead, we decided to use only the precisely detected directional derivatives and to modify the algorithm of watershed transformation accordingly.

Our modification of watershed transformation can be described as follows: water can not flood topographical surface in all directions, but only in directions where partial derivatives are computed. Such modification of watershed transformation allows optimal usage of the values of the first-order directional derivatives, as it employs the exactly known data only. Simple analysis shows that the result of application of our method to the segmentation of large objects is similar to that performed according to the traditional watershed transformation algorithm. On the contrary, the results of segmentation of smaller objects improve significantly, when our modification of watershed transformation is applied. The proposed approach is described in detail in [11].

\subsection{Description of the proposed algorithm}

The scheme of the proposed method is shown on figure 2. During the first stage of the proposed algorithm edge detection of small objects is executed. It consists of: (1) Application of a special gradient operator; (2) Application of a modification of watershed transformation for segmentation of small objects. After execution of first stage the positions of edges $E=\left\{e_{i}\right\}$ is saved for using at next stages. The following actions are executed cyclically with the in- 


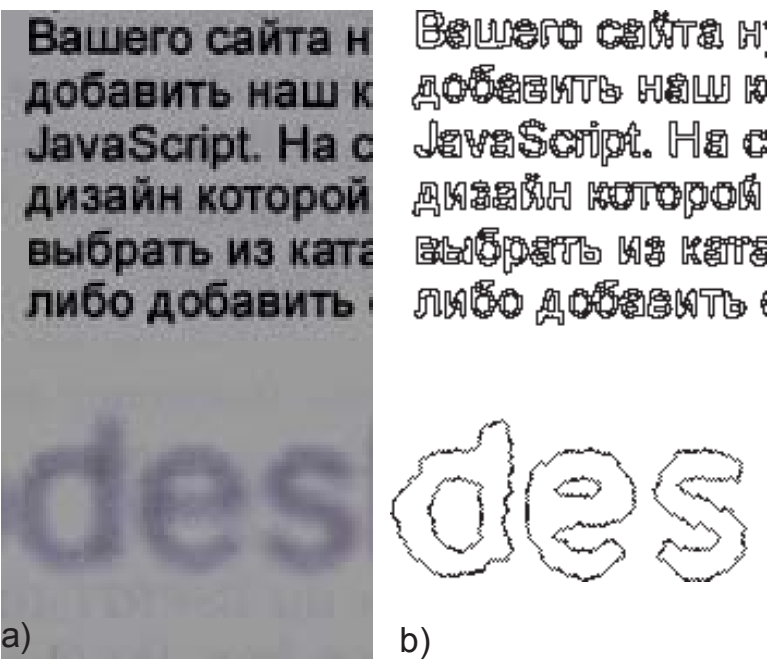

Figure 4. a) Original image with resolution $174 \times 304$

b) Result of segmentation using proposed method

crease of the scale value $s$ at each iteration until this value achieves the maximum scale value $s$

In each $i$ iteration of the cycle the following actions are executed:

(1) Construction of $s_{i}$-scale gradient image $G_{i}$ is executed taking into account positions of edges $E_{i-1}=\left\{e_{i-1}\right\}$ detected at the previous step $i-1$. As was mentioned above, computation of the gradient is executed at each point of the original image except at points that belong to $s_{i}$-neighbourhood of edge pixels $e_{i-1}$ detected at the previous step.

(2) Application of watershed algorithm.

(3) Saving of the detected at step (2) edge positions $E_{i}=\left\{e_{i}\right\}$ for using them at next iteration of the cycle. The method will result in detection of the final edge positions $E_{f}=\left\{e_{f}\right\}$ of the segmented regions.

\section{Experimental results}

The introduced method was probed on a large amount $(>1000)$ of images from color newspapers and magazines, and yields successful segmentation results. Examples of segmentation of color images using the proposed method are shown on figures 3-4. Image on figure 3 has resolution $306 \times 155$. Image on figure 4 has resolution $174 \times 304$. Example on figure 3 shows that the proposed method gives successful results for small objects sized up to 1 pixel. Image on figure 4 shows that the method allows successful segmentation of both objects with step edges and objects with ramp edges.

\section{Conclusion}

As experimental results show, the proposed method can be successfully used for segmentation of color im- ages. During the development of the introduced method we gave special attention to the segmentation of images from color newspapers and magazines. Acceptable results for such images give possibility to use method in OCR systems. The watershed transformation algorithm is the basis of the proposed method. Successful segmentation of small objects, such as letters or symbols, is achieved by using a special gradient operator and a modification of watershed transformation. Successful segmentation of both objects with step edges and objects with ramp edges is achieved by means of the analysis of gradient images of different scales. This multiscale analysis uses a fineto-coarse strategy and prevents the already detected sharp edges from smoothing while moving to coarser scales. The introduced algorithm allows in the same time to detect successfully ramp edges at coarse scales. The drawback of the proposed method is its significant computational time in case of a large amount of the processed scales. Reduction of the computational time is the subject of our current investigation.

\section{References}

[1] H.D. Cheng, X.H. Jiang, Y. Sun, Jingli Wang, "Color image segmentation: advances and prospets", Pattern Recognition 34, 2001, pp. 2259-2281.

[2]S. Beucher, F. Meyer, "The Morphological Approach to Segmentation: The Watershed Transformation", in Mathematical Morphology in Image Processing, E. R. Dougherty Editor, Marcel Dekker, Inc, New York, 1992, pp.433-481.

[3] R.C. Gonzalez, R.E.Woods, Digital Image Processing, PrenticeHall, Inc, Upper Saddle River, New Jersey, 2002, pp. 617-626.

[4] L. Vincent, P. Soille, "Watersheds in Digital Space: An Efficient Algorithm Based on Immersion Simulations", IEEE Trans. Pattern Anal. Mach. Intell 13 (6), 1991, pp. 583-598. [5] A. Bieniek, A. Moga, "An efficient watershed algorithm based on connected components", Pattern Recognition 33, 2000, pp. 907-916.

[6] D. Ziou, S. Tabbone, "Edge Detection Techniques - An Overview", technical report, No. 195, Dept Math \& Informatique. Universit de Sherbrooke, 1997.

[7] M. Kazanov, "One approach to multi-scale edge detection", Proceedings of the 3rd IASTED International Conference VIIP, Benalmadena, Spain, 2003, pp. 356-360.

[8] D.J.Williams and M.Shas, "Edge Contours Using Multiple Scales", Computer Vision, Graphics and Image Processing, 51, 1990, pp. 256-274.

[9] L.G. Roberts, "Machine Perception of Three-Dimensional Solids", Optical and Electro-Optical Information Processing, Tippet, J.T. (ed.), MIT Press, Cambridge, 1965.

[10] J.M.S. Prewitt, "Object Enhancement and Extraction", Picture Processing and Psychopictorics, Lipkin, B. S., and Rosenfeld, A. (eds.), Academic Press, New York, 1970.

[11] M.Kazanov, "Modification of Watershed Transformation for Images, Containing Small Objects", 17th International Conference on Pattern Recognition, Cambridge, UK, 2004. 